\title{
Development of CFRP mirrors for space telescopes
}

\author{
Shin Utsunomiya*a, Tomohiro Kamiya ${ }^{\mathrm{a}}$, Ryuzo Shimizu ${ }^{\mathrm{a}}$ \\ aJapan Aerospace Exploration Agency, 2-1-1 Sengen, Tsukuba, Ibaraki, Japan 305-8505
}

\begin{abstract}
CFRP (Caron fiber reinforced plastics) have superior properties of high specific elasticity and low thermal expansion for satellite telescope structures. However, difficulties to achieve required surface accuracy and to ensure stability in orbit have discouraged CFRP application as main mirrors. We have developed ultra-light weight and high precision CFRP mirrors of sandwich structures composed of CFRP skins and CFRP cores using a replica technique. Shape accuracy of the demonstrated mirrors of $150 \mathrm{~mm}$ in diameter was $0.8 \mu \mathrm{m}$ RMS (Root Mean Square) and surface roughness was $5 \mathrm{~nm}$ RMS as fabricated. Further optimization of fabrication process conditions to improve surface accuracy was studied using flat sandwich panels. Then surface accuracy of the flat CFRP sandwich panels of $150 \mathrm{~mm}$ square was improved to flatness of $0.2 \mu \mathrm{m}$ RMS with surface roughness of $6 \mathrm{~nm}$ RMS. The surface accuracy vs. size of trial models indicated high possibility of fabrication of over $1 \mathrm{~m}$ size mirrors with surface accuracy of $1 \mu \mathrm{m}$. Feasibility of CFRP mirrors for low temperature applications was examined for JASMINE project as an example. Stability of surface accuracy of CFRP mirrors against temperature and moisture was discussed.
\end{abstract}

Keywords: CFRP, mirror, space telescope, low temperature, surface preciseness, dimensional stability, CTE, CME

\section{INTRODUCTION}

Requirement of resolution for space telescopes has recently become severer and severer in order to observe deep space or to investigate precise astrometric positions of far stars. Enlarging aperture diameter of main mirrors is one of straight solutions to achieve high resolution, but satellites usually have very strict mass limit and larger mirrors must have lighter density solution. CFRP (carbon fiber reinforced plastics), which are used as main structures of both satellite and onboard mission sensors, are the most promising materials for main mirrors because of their physical properties of ultralight weight, low thermal expansion, high elasticity and high thermal conductivity in addition to manufacturability of large scale structures. However, space telescope mirrors are still commonly made of glasses or metals, e.g. ULE®, Zerodur ${ }^{\circledR}$, fused silica and beryllium [1]. Reasons why CFRP have not been used are because dimensional accuracy of $\mathrm{nm}$ level has not been achieved by their fiber print-through and long-term dimensional stability has not been characterized yet.

We have developed all CFRP mirrors of sandwich structures [2]. CFRP face skins and CFRP honeycomb cores were made of high modulus pitch carbon fibers and cyanate ester resin matrix. Carbon fibers and volume fraction and laminated constitution were selected to have nearly zero coefficient of thermal expansion (CTE) smaller than $1 \times 10^{-7} / \mathrm{K}$. Dimensional stability against temperature changes in orbital environments is primary concern in structure designs of space telescopes. For applications in cryogenic temperatures, CFRP possess the lowest thermal expansion in candidate materials, not only in service temperature but also between ambient to cryogenic temperatures [3]. Surface accuracy of $\mathrm{nm}$ level of the mirrors was achieved by using a replica technique [4]. Surface accuracy of optically polished glass tool was replicated to the CFRP mirror surface by thin epoxy resin layer deposition. Fig.1 shows an example of demonstration mirrors. Overall dimensional accuracy of the spherical mirrors of $150 \mathrm{~mm}$ in diameter was $0.8 \mu \mathrm{m}$ RMS (Root Mean Square) and surface roughness was $5 \mathrm{~nm}$ RMS as fabricated.

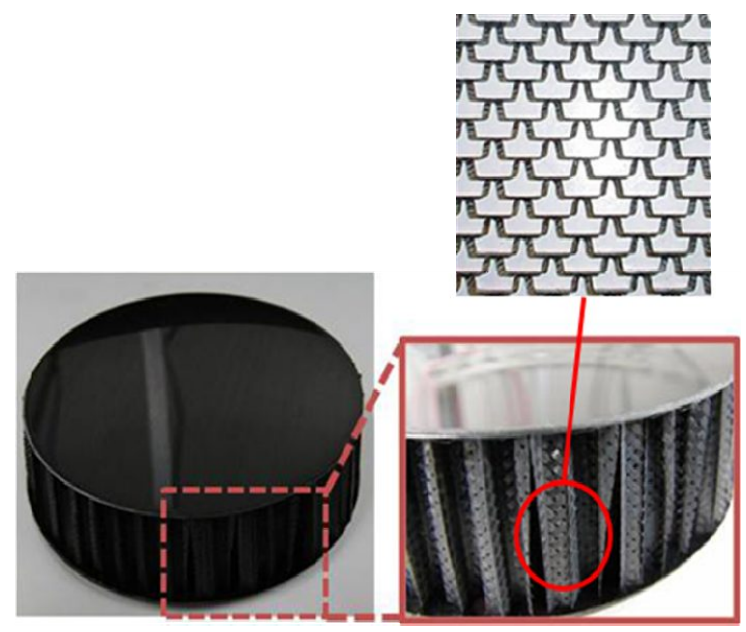

Fig.1 All CFRP mirrors

Ultra-low areal density High elastic modulus Very low CTE

$$
\begin{aligned}
& <7 \mathrm{~kg} / \mathrm{m}^{2} \\
& >100 \mathrm{GPa} \\
& <1 \times 10^{-7} / \mathrm{K}
\end{aligned}
$$


Feasibility of CFRP mirrors was examined for JASMINE Project [5] as an example. Telescope of JASMINE will be designed to work for wavelength of $1.7 \mu \mathrm{m}$ and at $210 \mathrm{~K}$ in orbit.

\section{JASMINE PROJECT}

JASMINE is abbreviation of Japan Astrometry Satellite Mission for Infrared Exploration [5]. Basic designs of SmallJASMINE and technical problems have now been investigated mainly at JASMINE project office of National Astronomical Observatory of Japan (NAOJ) in collaboration with JAXA, Kyoto University and other institutes. The main science objective is to clarify the structure formation model of the Galactic bulge, star formation histories around the Galactic center. Small-JASMINE will observe stars of a region around the Galactic center in an infrared Hw-band $(1.7 \mu \mathrm{m})$. JASMINE will determine positions and parallaxes accurate to $10 \mu$ arcseconds for millions of stars around the Galactic center brighter than $\mathrm{Hw}=11.5 \mathrm{mag}$. The survey will be planned to use a single beam telescope of which the diameter of the primary mirror is $300 \mathrm{~mm}$. Requirements for the main mirror will be $\lambda / 20$ in shape accuracy, $0.8 \mu \mathrm{m} P V$ and $\lambda / 50$ in surface roughness, $30 \mathrm{~nm}$ PV and to keep these shapes at the service temperature $210 \mathrm{~K}$ in low-earth orbit. Availability of CFRP mirrors were examined for requirements of the main mirror of JASMINE telescope.

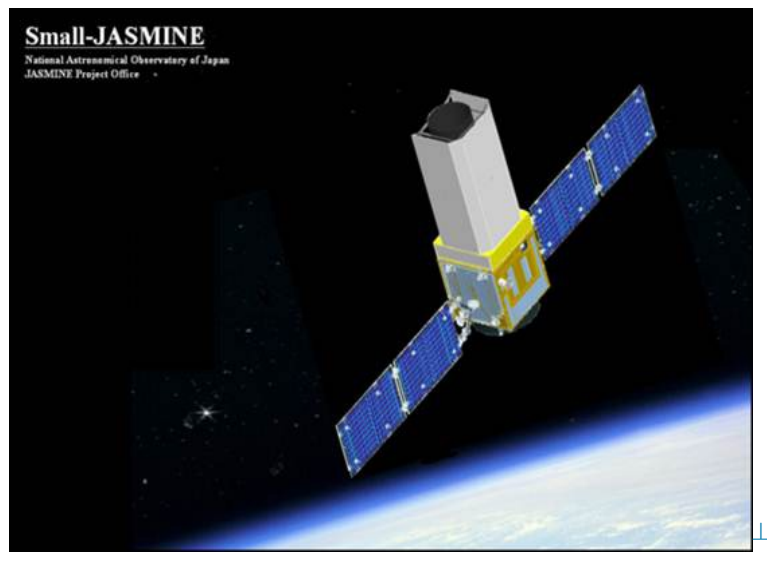

Fig.2 JASMINE

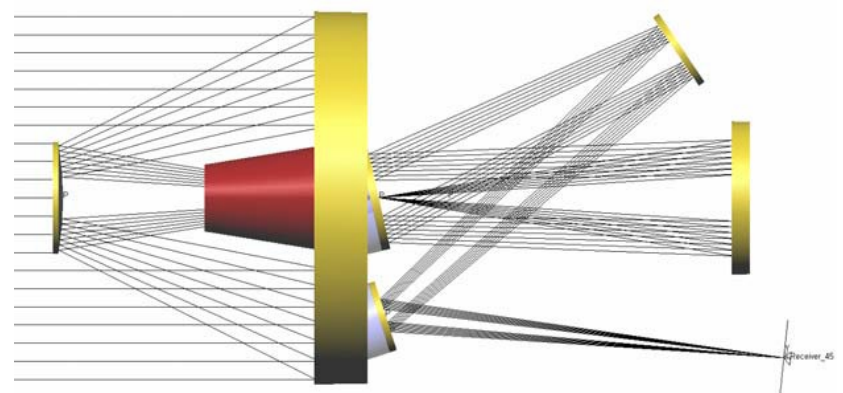

Fig.3 Telescope of JASMINE

\section{ALL CFRP MIRRORS AND EXPERIMENTAL PROCEDURE}

\subsection{CFRP mirror fabrication process}

The CFRP mirrors were composed of sandwich panels with CFRP face skins and CFRP flex cores (Fig.1). High modulus pitch carbon fibers YSH-60A (Nippon Graphite Fiber) and low moisture expansion resins of cyanate ester NM31 (Nippon Graphite Fiber) as matrix were used for face skins. Unidirectional prepregs were laminated in quasi-isotropic stack $[0 / \pm 45 / 90] 2 \mathrm{~s}$ of 16 plies and then pre-cured in an autoclave. Skin thickness was $1.7 \mathrm{~mm}$. The flex cores were also composed of high modulus pitch carbon fiber YSH-50A (Nippon Graphite Fiber) and cyanate ester resin matrix RS-3 (TenCate). Cores were made of plain-cloth prepreg mono-sheet. Fiber direction was $45^{\circ}$ to core height direction. Core size was $3 / 8 "(25.4 \mathrm{~mm})$ and core height was $40 \mathrm{~mm}$. Flex cores were selected for easy forming of a curved shape. Density of the cores was $30 \mathrm{~kg} / \mathrm{m}^{3}$. Pre-cured face sheets and cores were bonded using epoxy film adhesive. Layup tools of low expansion glass which were optical-polished to surface accuracy of $\lambda / 20(\lambda=632.8 \mathrm{~nm})$ were used for both face sheet curing and sandwich bonding. Diameter of the mirrors was $150 \mathrm{~mm}$ and thickness, $44 \mathrm{~mm}$ and concave surface curvature, $454 \mathrm{~mm}$. Arial densities of the mirrors were $6 \mathrm{~kg} / \mathrm{m}^{2}$. Flat CFRP sandwich panels of the same composition were also fabricated to investigate effects of fabricating process conditions on surface accuracy.

Surface of the front face skins of sandwich panels was deposited with epoxy resin using the same glass tool by a replica technique in order to get high surface accuracy and smooth surface.

\subsection{Experimental procedure}

Surface profiles of CFRP mirrors were observed using a laser interferometer (Zygo). Mirrors were set in a space 
chamber and laser beam was introduced through quartz window (Fig.4). A 3D optical profiler of white light interferometer (New View 7300, Zygo) was used to measure surface accuracy of flat CFRP specimens.

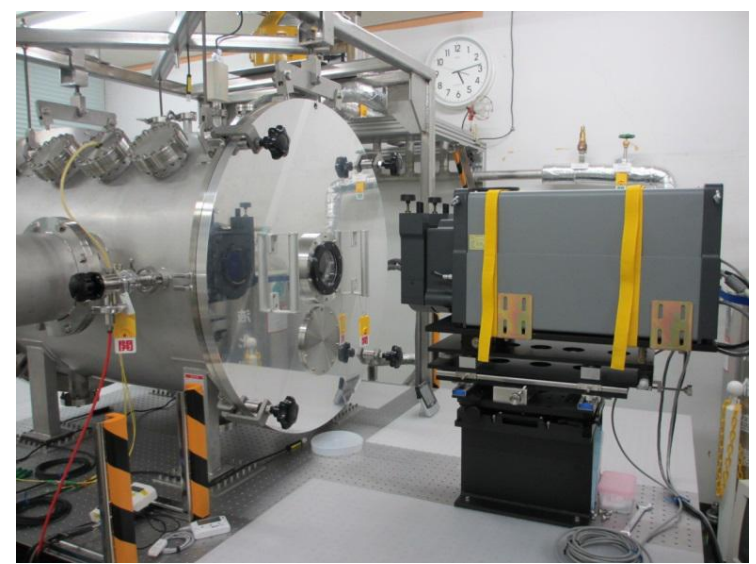

a. Space chamber and laser interferometer

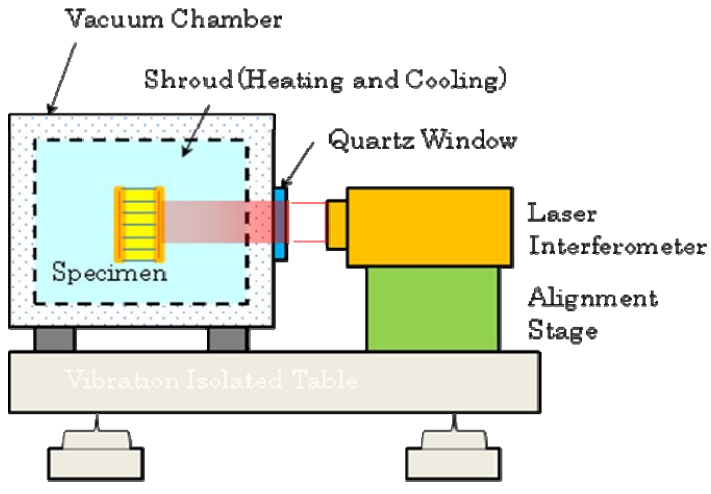

b. Schematic view of the apparatus

Fig. 4 Space chamber for observation of thermal deformation of mirrors

\section{STABILITY AGAINST TEMPERATURE AND MOISTURE}

Fig. 5 shows CTE of candidate materials for the main mirror. Zero expansion glasses or ceramics have very low CTE from 270 to $320 \mathrm{~K}$. Only CLEACERAM (Ohara) was shown as an example in Fig.5. CTE of CFRP is designable to have zero CTE at service temperatures and superior to the other materials. In addition, CTE of CFRP was almost flat within $1 \times 10^{-7} / \mathrm{K}$ in relatively wide range of temperatures. CTE of synthetic silica crosses zero at $180 \mathrm{~K}$ and it is thought to be promising.

Cyanate esters were selected as matrix resin for CFRP mirrors because they have low moisture absorption characteristics compared with epoxy resins. Fig. 6 shows moisture absorption rate of CFRP in aging at $60{ }^{\circ} \mathrm{C}, 90 \% \mathrm{RH}$ (Relative Humidity). Saturated moisture content of CFRP of cyanate matrix was $0.45 \%$ much lower than $1.2 \%$ of epoxy matrix. Fig.7 shows moisture expansion measured by FBG (Fiber Bragg Grating) sensors embedded in CFRP [6]. Moisture contents were measured with other samples of the same CFRP. CME (Coefficient of Moisture Expansion) was estimated to $2.2 \times 10^{-4} / \%$. This value was consistent with $\mathrm{CME}$ that was measured using a dilatometer in aging at $120{ }^{\circ} \mathrm{C}$ in vacuum (Fig.8). CFRP of cyanate matrix usually have $0.1 \sim 0.2 \%$ of moisture content while they are stored in a clean room condition of 20 to $25^{\circ} \mathrm{C}$ and 50 to $60 \% \mathrm{RH}$. Moisture desorption shrinkage in orbit would be estimated to 2 to $4 \mathrm{x}$ $10^{-5}$ in strain and that is several times larger than thermal distortion of $8 \times 10^{-6}$ from ground, $300 \mathrm{~K}$ to space $210 \mathrm{~K}$.

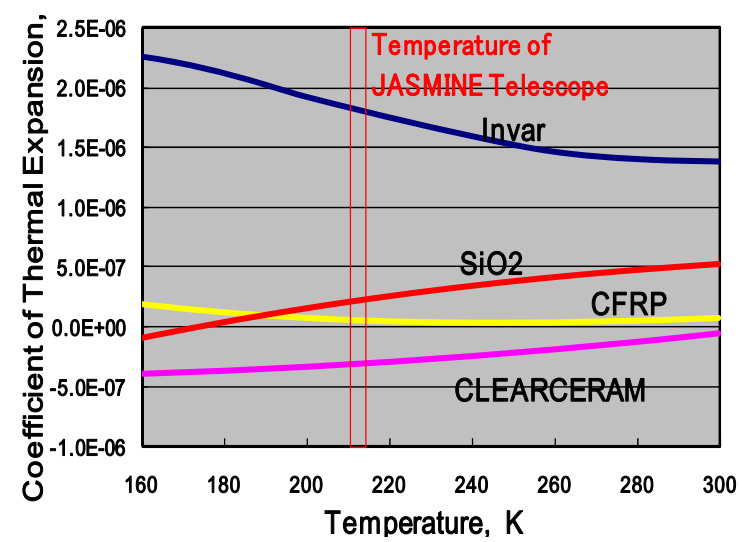

Fig.5 CTE of candidate materials for JASMINE mirror

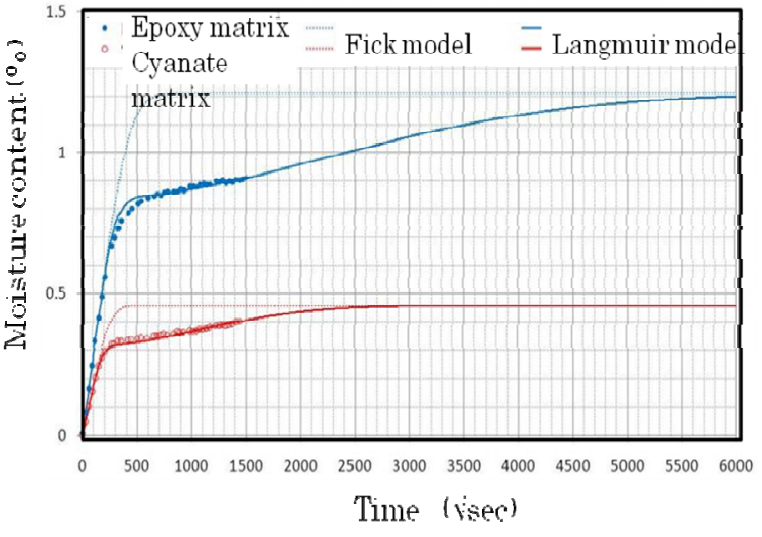

Fig. 6 Moisture absorption of CFRP 
Shape deformation of CFRP mirrors due to moisture expansion was observed to be similar with thermal distortion [2]. It is noted that shape accuracy of CFRP mirrors must be estimated in dried condition

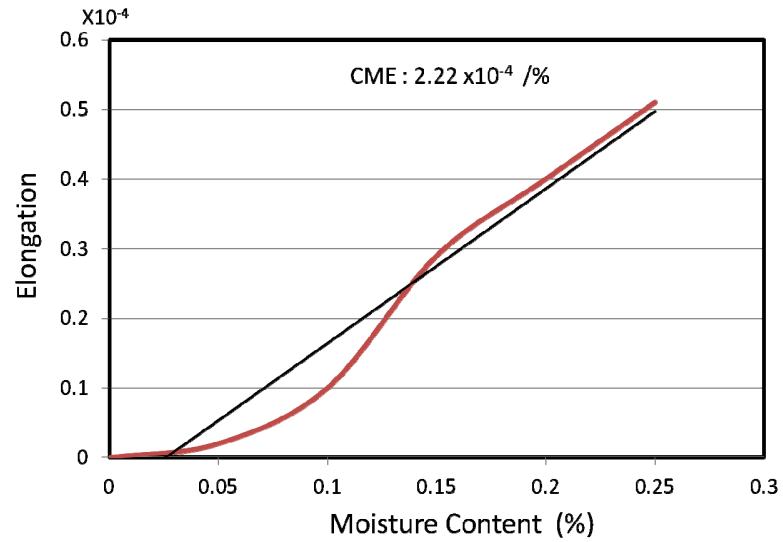

Fig.7 CME of CFRP at $60^{\circ} \mathrm{C} 90 \% \mathrm{RH}$

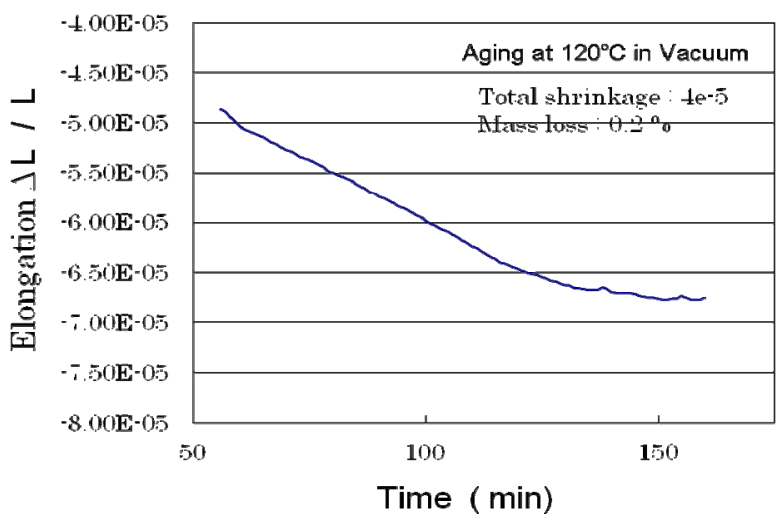

Fig. 8 Moisture desorption shrinkage at $120^{\circ} \mathrm{C}$ in vacuum

\section{SURFACE ACCURACY ACHIEVED BY REPLICA METHOD}

Shape accuracy of demonstrated mirrors of $150 \mathrm{~mm}$ in diameter was $0.8 \mu \mathrm{m}$ RMS and the surface roughness was $5 \mathrm{~nm}$ RMS as fabricated [4]. Shape accuracy of mirrors of $300 \mathrm{~mm}$ diameter was $8 \mu \mathrm{m}$ PV (Peak to Valley) and not accurate enough for the JASMINE requirement of $0.8 \mu \mathrm{m}$ PV. Improvement of shape accuracy by optimization of fabrication process conditions was studied [7]. Fig.9 shows surface accuracy of flat sandwich panels of $150 \times 150 \mathrm{~mm}$ square in accordance with measurement areas. The surface accuracy was improved to $0.2 \mu \mathrm{m}$ RMS in $100 \mathrm{~mm}$ square area, and dependency on measurement areas became significantly small. Extrapolation of the data in Fig.9 indicated that shape accuracy of mirrors of $1 \mathrm{~m}$ diameter would be less than $1 \mu \mathrm{m}$ RMS.

Shape accuracy was predominantly determined by overall warping or twisting. To improve shape accuracy, it is critical and important to reduce the overall warping. There were existed two types of overall warping, spherical and saddleshaped (Fig.10). Spherical deformation was caused by inhomogeneous heating and cooling in curing process, especially temperature gradient in thickness direction. Saddle-shape deformation was occurred due to fiber misalignment [8]. Optimization of fabrication process conditions would reduce overall warping. Even heating and cooling of laminates in curing process, especially in a symmetric condition in thickness direction dramatically improved shape accuracy.

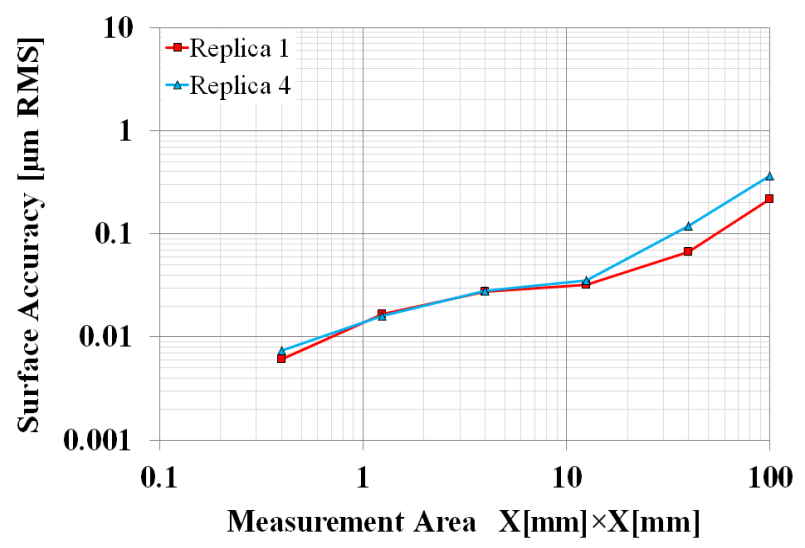

Fig.9 Surface accuracy of replicated CFRP skins of sandwich panels

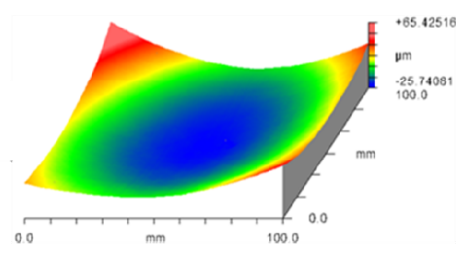

a. Spherical deformation

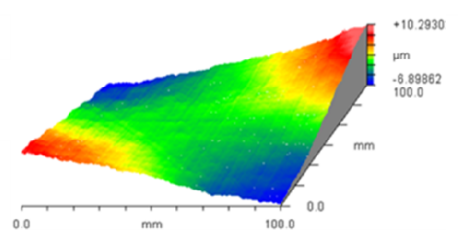

b. Saddle shape deformation

Fig.10 Overall deformation 
To reduce overall warping, heightening core thickness is one of effective methods in design, though areal density of sandwich panels rises.

Fiber tow patterns were another issue to degrade shape accuracy. In middle range of measurement areas of 10 to $100 \mathrm{~mm}$ square, undulation due to streaky patterns of fibers degraded shape accuracy (Fig.11). Replica removed fiber tow printthrough, but several $10 \mathrm{~nm}$ undulation remained. It might be necessary to improve homogeneity of prepregs in order to remove fiber tow print-through thoroughly.

Extrapolation of surface accuracy of demonstrated mirrors indicated feasibility of larger diameter mirrors of high shape accuracy. Estimated level of $1 \mu \mathrm{m}$ RMS is not accurate enough for the JASMINE main mirror though surface roughness was satisfied with the requirement. Optical machining was tried and shape accuracy better than $1 \mu \mathrm{m}$ PV was obtained at a trial basis.

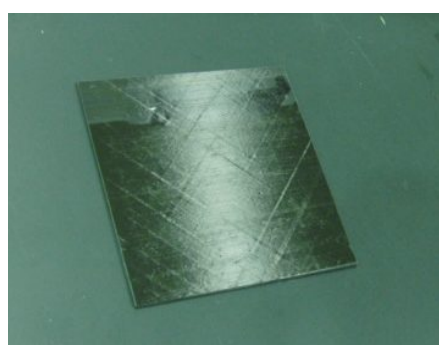

Fig.11 Streaky patterns of fiber tow before replica

\section{DEFORMATION IN TEMPERATURE CYCLE}

Fig.12 was the surface accuracy change when CFRP mirrors were cooled down from ambient to liquid nitrogen temperature [9]. Lines of upper two are surface accuracy change of the mirror in RMS. Lower two lines indicate difference from the original surface at room temperature. Shape accuracy happened to become better in low temperatures in this case. Difference of surface accuracy increased to 0.6 $\mu \mathrm{m}$ RMS towards $200 \mathrm{~K}$. 3-dimensional shapes at $300 \mathrm{~K}$ and $210 \mathrm{~K}$ was shown in Fig.13. Difference between $300 \mathrm{~K}$ and $210 \mathrm{~K}$ was $700 \mathrm{~nm}$ PV and $115 \mathrm{~nm}$ RMS. This number was not small in comparison with the required accuracy of $800 \mathrm{~nm}$ PV. It is concluded that shape accuracy of main mirrors must be measured at service temperature and confirmed to satisfy surface accuracy requirements.

Fig.14 shows surface profiles of the CFRP flat panels. Changes of overall warping were contrary in heating (a to b) and cooling (a to c). That suggested that deformation mechanism would be same between shape accuracy of asfabricated mirrors and their thermal deformation. Shape accuracy of as-fabricated mirrors was affected by temperature change from curing to room temperature. Improvement of shape accuracy of as-fabricated mirrors could improve dimensional stability in thermal cycling and deformation of temperature change and moisture absorption.

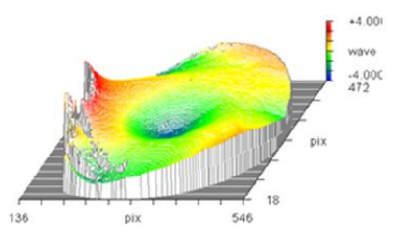

a. $298 \mathrm{I}$

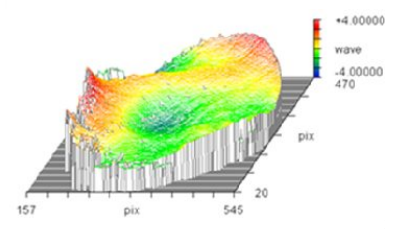

b. $20 \% \mathrm{~K}$

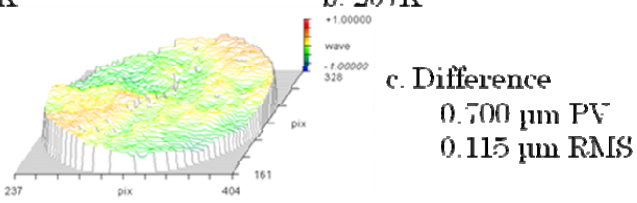

Fig.13 Difference between 300K and 210K

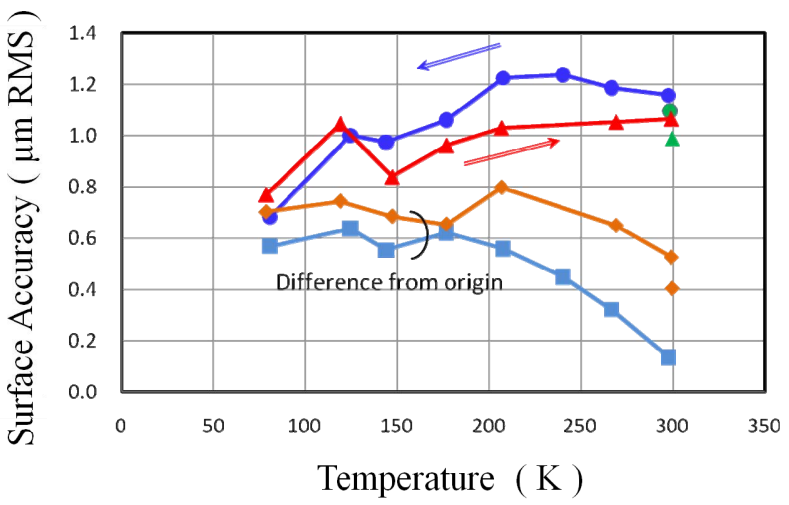

Fig.12 Change of surface accuracy of CFRP mirror in low temperatures

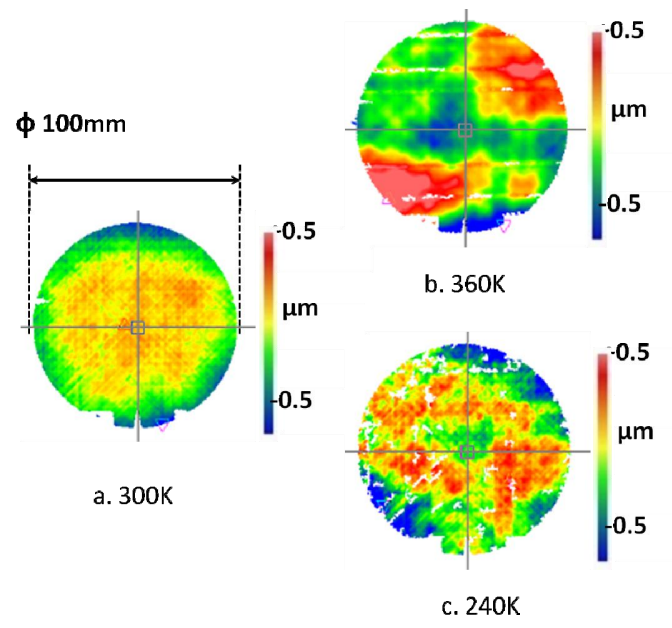

Fig.14 Thermal deformation of flat CFRP panels 


\section{CONCLUSION}

Ultra-lightweight and high-accuracy CFRP mirrors for space telescopes were fabricated using a replica technique. The CFRP mirrors were composed of sandwich panels with CFRP skins and CFRP cores. Shape accuracy of the demonstrated mirrors of $150 \mathrm{~mm}$ in diameter was $0.8 \mu \mathrm{m}$ RMS and surface roughness was $5 \mathrm{~nm}$ RMS as fabricated. Areal density was achieved to $7.0 \mathrm{~kg} / \mathrm{m}^{2}$. Availability of CFRP mirrors were evaluated for application to the main mirror of Small-JASMINE telescope. Shape accuracy was not good enough to the requirement of $800 \mathrm{~nm} \mathrm{PV}$. After optimization of fabrication process conditions shape accuracy was improved to $0.2 \mu \mathrm{m}$ RMS and surface roughness, 6.2 $\mathrm{nm}$ RMS. Measurement apparatus for thermal test in a vacuum chamber with a He-Ne laser interferometer was constructed and the demonstrated mirrors were tested at cryogenic temperature. Thermally induced figure error was 700 $\mathrm{nm}$ PV and $115 \mathrm{~nm}$ RMS at $210 \mathrm{~K}$, operating temperature of JASMINE telescope. CTE and CME of CFRP for mirrors were also measured. The CTE of CFRP were superior to other candidate materials. Deformation due to moisture desorption shrinkage in orbit would be estimated to be several times larger than thermal distortion due to temperature change from ground to orbit.

It was concluded that further improvement of shape accuracy and thermal stability of CFRP mirrors were necessary. It was thought critical and important to get highly dimensionally accurate mirrors as cured condition in order to reduce dimensional degradation in low temperatures. Temperature change was a driving force to cause change of shapes in both cases. There were two types of overall warping, spherical and saddle shapes. Fiber misalignment caused saddle-shape deformation. Temperature gradient to thickness direction in curing process caused spherical-shape deformation. Improvement of fabrication process would reduce overall warping. Streaky patterns of fiber tows were another issue which degraded shape accuracy. Homogeneity of prepregs must be improved to reduce undulation. Optical machining and polishing to get shape accuracy was residual issue for future work.

\section{REFERENCES}

[1] D. Baiocchi and H. P. Stahl "Enabling Future Space Telescopes: Mirror Technology Review and Development Roadmap". Astro2010: The Astronomy and Astrophysics Decadal Survey, Technology Development Papers, no. 23, (2009)

[2] Utsunomiya S., Kamiya T., Shimizu R., "CFRP composite mirrors for space telescopes and their microdimensional stability”, Proc. SPIE, San Diego U.S.A., 7739-116, (2010)

[3] Chen P.C., Bowers C.W., Content D.A., Marzouk M., Romeo R., Advances in Very Lightweight Composite Mirror Technology, Opt. Eng., 39(9), 2320-2329, (2000)

[4] Kamiya T., Utsunomiya S., Shimizu R., Improvement of CFRP mirrors by replica method , Proc. ICCM-18, Jeju Island Korea, AF1018, (2011)

[5] JASMINE home page, www.jasmine-galaxy.org

[6] S. Utsunomiya and R. Shimizu "Monitoring of Dimensional Stability of CFRP Mirrors for Space Telescopes by Using Embedded FBG Sensors". Proceedings of the 17th International Conference on Composite Materials, Edinburgh England, IA2:4,( 2009)

[7] Utsunomiya S., Kamiya T., Shimizu R., Development of CFRP Mirrors for space telescopes, Proc. SAMPE2013, San Diego U.S.A., 3191, (2013)

[8] Arao Y., Koyanagi J., Utsunomiya S., Kawada H., Effect of ply angle misalignment on out-of-plane deformation of symmetrical cross-ply CFRP laminates: Accuracy of the ply angle alignment, Composite Structures, 93, 1225-1230, (2011)

[9] Utsunomiya S., Kamiya T., Shimizu R., Dimensional Stability of CFRP Mirrors for space telescopes under low temperature environment, Proc. ICCM-18, Jeju Island Korea, AF0933, (2011) 